\title{
TAMING CODIMENSION THREE EMBEDDINGS ${ }^{1}$
}

\author{
BY T. B. RUSHING ${ }^{2}$
}

Communicated by Richard D. Anderson, January 27, 1969

1. Taming embeddings of certain polyhedra. If $P \subset K$ are polyhedra, we say there is an elementary $C(X)$-construction from $P$ to $K$, written $P^{C_{0}(X)} \nearrow K$, if there exists a pair $(\mathfrak{C}(\chi), \chi)$ which is PL homeomorphic to $(C(X), X)$, where $C(X)$ denotes the cone over a polyhedron $X$, such that

(1) $K=P \cup \mathfrak{e}(\chi)$,

(2) $\chi=P \cap \mathfrak{e}(\chi)$, and

(3) $P$ is link collapsible on $\chi$ and $\mathrm{Cl}(\mathrm{P}-\chi)=P$. A polyhedron $K$ is said to be $C(B)$-constructible from $P$, written $P^{C(B)} \nearrow K$, if there is a finite sequence, $P^{C_{e}\left(X_{1}\right)} \nearrow P_{1}^{C_{e}\left(X_{2}\right)} \nearrow P_{2}{ }^{C_{e}\left(X_{3}\right)} \nearrow \ldots C_{e}\left(X_{p}\right) \nearrow K$, of elementary constructions from $P$ to $K$ where each $X_{i}$ is a PL ball. Similarly, $K$ is said to be $C(S)$-constructible from $P$, written $P^{C(S)} \nearrow K$, if there is such a finite sequence where each $X_{i}$ is a PL sphere. A $P$-nice polyhedron $K$ has the property that $P^{C(B)} \nearrow L^{C(S)} \nearrow K$. A $B$-nice polyhydron, $B$ a ball, is simply called a nice polyhedron. An embedding $f$ of a polyhedron $P$ into a polyhedron $Q$ is isotopically tame if there is an isotopy $e_{t}$ of $Q$ such that $e_{0}=1$ and $e_{1} f$ is PL. The embedding $f$ is $\epsilon$-tame if for any $\epsilon>0$ there is an isotopy $e_{t}$ of $Q$ such that $e_{0}=1, e_{1} f$ is PL, dist $\left(x, e_{t}(x)\right)<\epsilon$ for all $x \in Q$ and $e_{t} \mid Q-N_{\epsilon}(f(P))=1$.

Theorem 1. Let $K$ be a $P$-nice polyhedron such that $\operatorname{dim}(\mathrm{Cl}(K-P))$ $\leqq n-3, n \geqq 5$, and let $f: K \rightarrow Q^{n}$ be an embedding of $K$ into the interior of the PL n-manifold $Q$ which is locally flat on the open simplexes of some triangulation and is such that $f \mid P$ is isotopically tame. Then, $f$ is isotopically tame and if $f \mid P$ is $P L$, the taming isotopy is fixed on $f(P)$.

TheOREM 2. If $f: I^{k} \rightarrow \dot{M}^{n}, n \geqq 5$, (no restriction on $k$ ) is a locally flat embedding of $I^{k}$ into the interior of the PL n-manifold $M$, then $f$ is $\epsilon-$ tame.

THEOREM 3. If $f: S^{k} \rightarrow \dot{M}^{n}, n \geqq 5, n-k \geqq 3$, is a locally flat embedding of $S^{k}$ into the interior of the PL $n$-manifold $M$, then $f$ is $\epsilon$-tame.

1 This research overlaps the author's doctoral dissertation which was written under the direction of Professor J. C. Cantrell at the University of Georgia. Some of the more recent results of this announcement (in particular the theorems concerning the taming of cells and spheres) are joint results with Professor Cantrell.

2 Partially supported by NSF grants GZ-45.1 and GP-6613. 
THEOREM 4. If $f: S^{n-1} \rightarrow S^{n}, n \geqq 5$, is a locally flat embedding and $\epsilon>0$, then there is an isotopy $e_{t}$ of $S^{n}$ such that

(1) $e_{0}=1$,

(2) $e_{1} f$ is $P L$, and

(3) dist $\left(x, e_{t}(x)\right)<\epsilon$ for all $x \in S^{n}$.

The next theorem follows directly from Theorem 1 and Theorem 2 .

TheOREM 5. Let $K$ be a nice polyhedron such that $\operatorname{dim}(\mathrm{Cl}(K-B))$ $\leqq n-3, n \geqq 5$, and let $f: K \rightarrow \stackrel{M}{ }^{n}$ be an embedding which is locally flat on the open simplexes of some triangulation. (If $\operatorname{dim} B>n-3$, we also require that $f$ be locally flat on $B$.) Then, $f$ is isotopically tame.

THEOREM 6. Let $f_{i}: I^{k_{i}} \rightarrow I^{n}, n-k_{i} \geqq 3, n \geqq 5, i=1,2, \cdots, r$ be proper embeddings such that

(1) $f_{i} \mid \dot{I}^{k_{i}}: \dot{I}^{k_{i}} \rightarrow \dot{I}^{n}$ is locally flat,

(2) $f_{i} \dot{I}^{k_{i}}$ is locally flat, and

(3) $f_{i}\left(I^{k_{i}}\right) \cap f_{j}\left(I^{k_{j}}\right)=\varnothing$ when $i \neq j$.

Then, for $\epsilon>0$ there is an isotopy $e_{t}: I^{n} \rightarrow I^{n}$ such that

(1) $e_{0}=1$,

(2) $e_{t} \mid I^{n}-N_{\mathrm{e}}\left(\mathrm{U}_{i=1}^{r}\left(f_{i}\left(I^{k_{i}}\right)\right)\right)=1$, and

(3) $e_{1} f_{i}: I^{k_{i}} \rightarrow I^{n}$ is $P L$ for $i=1,2, \cdots, r$.

Corollary 6.1. Spheres $S_{i}{ }^{k_{i}}, i=1,2, \cdots, r$, contained locally flatly in $S^{n}, n-k_{i} \geqq 3, n \geqq 5$, are unlinked if and only if regarding $S^{n}$ as $i^{n+1}, S^{k_{i}}, i=1,2, \cdots, r$ bound disjoint cells $D^{k_{i+1}}, i=1,2, \cdots r$, respectively, whose interiors are contained in the interior of $I^{n+1}$ and are locally flat there.

The above corollary follows from Theorem 7 of [5].

2. Taming PL manifolds around the boundary. A topological embedding $f: M^{k} \rightarrow Q^{n}$ of the PL $k$-manifold $M$ into the PL $n$-manifold $Q$ is said to be allowable if $f^{-1}(\dot{Q})$ is a PL $(k-1)$-submanifold (possibly empty) of $\dot{M}$.

TheOREM 7. Let $f: M^{k} \rightarrow Q^{n}, n-k \geqq 3, n \geqq 5$, be an allowable embedding of the $P L k$-manifold $M$ into the $P L n$-manifold $Q$ such that $f \mid f^{-1}(\dot{Q})$ is $P L$ and $f \mid\left(M-f^{-1}(\dot{Q})\right)$ is locally flat and let $\epsilon>0$ be given. Then, there is a neighborhood $u$ of $f^{-1}(\dot{Q})$ in $M$ and an $\epsilon$-push $e_{t}$ of $\left(Q, f\left(f^{-1}(\dot{Q})\right)\right)$ which is fixed on $\dot{Q}$ such that $e_{1} f \mid \mathcal{u}: \mathcal{u} \rightarrow Q$ is $P L$.

3. Some conjectures. Let $P$ be a polyhedron contained in the PL ball $H$. Then, an embedding $f: P \cup \stackrel{\circ}{H} \rightarrow Q^{n}$ of $P \cup \dot{H}$ into the interior of the PL manifold $Q$ is said to be piecewise linear (PL) if for every 
regular neighborhood $N$ of $\dot{H} \bmod P$ in $H$ (in the sense of [3]) it is true that $f \mid \mathrm{Cl}(H-N)$ is $P L$ in the usual sense.

Conjecture $\eta^{1}(n, k, p)$. Let $D^{n} \subset E^{n}$ be a locally flat $n$-cell, let $P^{p} \subset I^{k}$ be a polyhedron and let $f: I^{k} \rightarrow D$ be a proper, locally flat embedding such that $f \mid P$ is $P L$. Then, there is a proper embedding $g: I^{k} \rightarrow D$ such that

(1) $g\left|\left(P \cup \dot{I}^{k}\right)=f\right|\left(P \cup \dot{I}^{k}\right)$, and

(2) $g \mid\left(P \cup \dot{I}^{k}\right)$ is $P L$.

Conjecture $\eta^{2}(n, k, m)$. Let $D^{n} \subset E^{n}$ be a locally flat $n$-cell and let $R$ and $R^{\prime}$ be regular neighborhoods of $\stackrel{0}{I}^{m} \times I^{k-m}$ in $I^{k}$ such that $R^{\prime}$ is contained in the point-set interior of $R$. Suppose that $f: I^{k} \rightarrow D$ is a proper, locally flat embedding such that $f \mid R$ is PL. Then, there is a proper embedding $g: I^{k} \rightarrow D$ such that

(1) $g\left|\left(R^{\prime} \cup \dot{I}^{k}\right)=f\right|\left(R^{\prime} \cup \dot{I}^{k}\right)$, and

(2) $g \mid\left(R^{\prime} \cup I^{k}\right)$ is $P L$.

Of course the above conjectures are true whenever $k<n / 2$ simply by general position. It follows easily by techniques of Homma that $\eta^{2}$ is true whenever $k \leqq(2 / 3) n-1$ and it follows from Berkowitz's modification of those techniques announced in [1] that $\eta^{2}$ is true whenever $k<(3 / 4) n-1$. However, this conjecture has such a simple setting that it should follow in codimension three by a more straightforward approach.

Proposition 1. $\eta^{1}(n, k,-1)=\eta^{2}(n, k, 0)$ is true for $n-k \neq 2, n \geqq 5$, and $\eta^{2}(n, k, k)$ is true for $k \leqq n-3$.

ConJeCtURE $\omega(n, k)$. Let $M^{k} \subset E^{n}, n-k \geqq 3$, be a (possibly infinite) $k$-dimensional $P L$ manifold. Suppose that $f: E^{n} \rightarrow E^{n}$ is a (topological) homeomorphism such that $f \mid M^{k}$ is $P L$. If $\epsilon(x)>0$ is a continuous function on $E^{n}$, then there is a PL homeomorphism $g: E^{n} \rightarrow E^{n}$ which is an $\epsilon(x)$-approximation of $f$ such that $g|M=f| M$.

Bing [2] and Connell [4] show that $\omega(n, k)$ is true whenever $M=\varnothing$, i.e., $\omega(n,-1)$ is true. Also it should be pointed out that Siebenmann and Sondow [7] have shown that for $n \geqq 5$, there is a $\mathrm{PL}(n-2)$-sphere $K$ in $S^{n}$ and a (topological) homeomorphism $h: S^{n} \rightarrow S^{n}$ such that $h \mid K$ is PL and for which there is no PL homeomorphism $g: S^{n} \rightarrow S^{n}$ such that $g|K=h| K$. However, $K$ is not locally flat in $S^{n}$ in these examples.

Proposition 2. $\omega(n, k) \Rightarrow \eta^{2}(n, k, m), m=0,1, \cdots, k$, for $n-k \neq 2$.

Conjecture $\tau^{1}(n, k, p)$. Let $M^{k}$ and $Q^{n}$ be PL manifolds and suppose 
that $P^{p} \subset M^{k}, p \leqq k$, is a polyhedron. Let $f: M \rightarrow Q$ be an embedding such that $f \mid P$ and $f \mid(M-P)$ are $P L$. Then, $f$ can be $\epsilon$-tamed by an isotopy which is the identity outside the $\epsilon$-neighborhood of $f(P)$.

The following theorem is a special case of $\tau^{1}$. (Actually we have proved a result slightly more general than Theorem 8 .)

Theorem 8. Let $M^{k}$ and $Q^{n}, n-k \geqq 3, n \geqq 5$, be PL manifolds and suppose that $P_{i}^{p_{i}}, i=1,2, \cdots, r$, are disjoint collapsible polyhedra (for instance balls) contained in $M$ such that either $P_{i} \cap \dot{M}=\varnothing$ or $P_{i} \cap \dot{M}$ is collapsible. Let $\epsilon>0$ be given. If $f: M \rightarrow \dot{Q}$ is an embedding such that $f \mid \cup_{i=1}^{r} P_{i}$ and $f \mid\left(M-\cup_{i=1}^{r} P_{i}\right)$ are $P L$, then $f$ is tame, and the taming isotopy is the identity outside the $\epsilon$-neighborhood of $f\left(\mathrm{U}_{i=1}^{r} P_{i}\right)$.

The final conjecture is weaker than $\tau^{1}$, however it does not follow from Theorem 8 .

Conjecture $\tau^{2}(n, k, m)$. Let $M^{k}$ be a $k$-dimensional $P L$ manifold. Suppose $M^{k}=M_{*}^{k} \cup H^{k}$ where $M_{*}^{k}$ is a $k$-dimensional $P L$ manifold and $\left(H^{k}, H^{k} \cap M_{*}^{k}\right)^{P L}\left(I^{k}, \dot{I}^{m} \times I^{k-m}\right)$ for some $m$ such that $0 \leqq m \leqq k$. Furthermore, suppose that $f: M \rightarrow \dot{Q}$ is an embedding such that $f \mid\left(M^{k}-\left(H^{k} \cap M_{*}^{k}\right)\right)$ and $f \mid M_{*}^{k}$ are PL. Then, $f$ can be $\epsilon$-tamed by an isotopy which is the identity outside the $\epsilon$-neighborhood of $f\left(H^{k} \cap M_{*}^{k}\right)$.

4. Taming locally flat embeddings of PL manifolds. Let $f: M^{k} \rightarrow Q^{n}, n-k \geqq 3, n \geqq 6$ (also $n=5$ if $f \mid f^{-1}(\dot{Q})$ is PL), be an allowable embedding of the PL manifold $M^{k}$ into the PL manifold $Q^{n}$ such that $f \mid f^{-1}(\dot{Q})$ and $f \mid\left(M-f^{-1}(\dot{Q})\right)$ are locally flat. Also, let $f \mid P$ be PL where $P$ is a subpolyhedron of $M$ such that $P \cap\left(M-f^{-1}(\dot{Q})\right)$ is compact.

THEOREM 9. Suppose that $\eta^{2}(n, k, m)$ and $\eta^{2}(n-1, k-1, m-1)$ are true for every $m$ such that $0 \leqq m \leqq k$. Also suppose that $P \cap f^{-1}(\dot{Q})$ is a $P L(k-1)$-manifold and $P \cap\left(M-f^{-1}(\dot{Q})\right)$ is a $P L k$-manifold. Then, $f$ can be $\epsilon$-tamed by an isotopy $e_{t}$ such that $e_{t} \mid P=1$ for all $t$.

The $\eta$-conditions of Theorem 9 may be replaced by $\omega(n, k)$ and $\omega(n-1, k-1)$ by Proposition 2 .

Theorem 10. Suppose $\eta^{1}(n, k, p)$ and $\eta^{1}(n-1, k-1, p-1)$ are true for every $p$ such that $0 \leqq p \leqq k$. Then, $f$ can be $\epsilon$-tamed by an isotopy $e_{t}$ such that $e_{1} f|P=f| P$.

Theorem 11. Suppose that $\tau^{2}(n, k, m)$ and $\tau^{2}(n-1, k-1, m-1)$ are true for every $m$ such that $0 \leqq m \leqq k$. Then, $f$ is $\epsilon$-tame. 
Addendum (February 4, 1969). The author has proved that $\eta^{2}(n, k, m)$ is true for $n-k \geqq 3$ and $n \geqq 5$. Hence, a general taming theorem holds in codimension three (see Theorem 9).

AdDED IN Proof. For $n-k \geqq 3$ and $n \geqq 5, \eta^{2}(n, k, m)$ can be established by an argument which uses Hudson's Concordance Theorem, the Stable Homeomorphism Theorem and Connell's Approximation Theorem. Also, $\eta^{2}(n, k, m)$ follows in codimension three by employing the Stable Homeomorphism Theorem, Connell's Approximation Theorem and techniques used by R. C. Kirby to smooth locally flat embeddings of differentiable manifolds in the metastable range. R. T. Miller adapted these techniques to the PL category. Recall that we pointed out in the text that $\eta^{2}(n, k, m)$ follows from techniques of $\mathrm{T}$. Homma for $k<(3 / 4) n-1$. Thus, in this range our taming theorem is independent of the recent work on the triangulation problem. J. I. Cobb has announced a result which imples $\tau^{1}(n, k, p)$ for $n-k \geqq 3$. By using Cobb's result, the Stable Homeomorphism Theorem and Connell's Approximation Theorem, one can prove that any embedding $f: P^{k} \rightarrow Q^{n}, n-k \geqq 3$, of a polyhedron $P$ into the interior of a PL manifold $Q$ which is locally flat on the open simplexes of some triangulation is $\epsilon$-tame. J. L. Bryant and C. L. Seebeck have shown that if an embedding of a PL manifold into another PL manifold is locally nice, then it is $\epsilon$-tame, in the metastable range. Their proof uses Homma's Approximation Theorem and Hudson's Concordance Theorem. While our taming theorem is weaker in this range since the locally nice hypothesis is replaced by a locally flat hypothesis, the proof is simple and avoids Hudson's Concordance Theorem. R. T. Miller independently proved a taming theorem in the metastable range for the case that the embedding is into the interior of the ambient manifold in his thesis. It should be pointed out that A. V. Cernavskii has a lengthy but clever proof of a taming theorem for embeddings of polyhedra into the interior of PL manifolds in the metastable minus one range. $R$. T. Miller has recently announced a proof of a codimension three taming theorem for embeddings of PL manifolds into the interior of the ambient manifold. Kirby and Siebenmann have announced a similar result along with a nice result in codimensions less than three. Kirby had previously and independently observed that a codimension three taming theorem with an additional stable hypothesis would follow in the case that the embedding is into the interior of the ambient manifold. I would like to thank Lacher for bringing this to my attention as well as the result of John Cobb mentioned above. Complete proofs of the results 
stated in this announcement as well as some generalizations will appear elsewhere.

\section{REFERENCES}

1. H. W. Berkowitz, Piecewise linear approximations of homeomorphisms, Notices Amer. Math. Soc. 15 (1968), 731.

2. R. H. Bing, Stable homeomorphisms on $E^{5}$ can be approximated by piecerwise linear ones, Notices Amer. Math. Soc. 10 (1963), 666.

3. M. M. Cohen, $A$ general theory of relative regular neighborhoods, Trans. Amer. Math. Soc. 136 (1969), 189-229.

4. E. H. Connell, Approximating stable homeomorphisms by piecewise linear ones, Ann. of Math. (2) 78 (1963), 326-338.

5. W. B. R. Lickorish, The piecewise linear unknotting of cones, Topology 4 (1965), 67-91.

6. T. B. Rushing, Taming embeddings of certain polyhedra in codimension three, Trans. Amer. Math. Soc. (to appear).

7. L. Siebenmann and J. Sondow, Some homeomorphic spheres that are combinatorially distinct, Comment. Math. Helv. 41 (1967), 261-272.

University of Georgia, Athens, Georgia 30601 